\title{
ON LANDAU SCENARIO OF CHAOTIZATION FOR BEAM DISTRIBUTION
}

\author{
Z. Parsa*, \\ Brookhaven National Laboratory \\ Physics Depart. 901A, Upton, NY 11973 \\ V. Zadorozhny, \\ Institute of Cybernetic \\ National Academy of Sciences of Ukraine.
}

\author{
PAC99 \\ New York City \\ March 29 ${ }^{\text {th }}$ - April 2 ${ }^{\text {nd }}, 1999$
}

* Supported by U.S. Department of Energy Contract Number DE-AC02-98CH10886. E-mail: parsa@bnl.gov. 


\title{
ON LANDAU SCENARIO OF CHAOTIZATION FOR BEAM DISTRIBUTION *
}

\author{
Z. Parsa, ${ }^{\dagger}$ Brookhaven National Laboratory, 901 A, Upton, NY 11973, USA \\ V.Zadorozhny ${ }^{\ddagger}$, Institute of Cybernetic National Academy of Sciences of Ukraine.
}

\begin{abstract}
We examine a problem in nonlinear dynamics in which both regular and chaotic motions are possible. Thus we deal with some of the fundamental theoretical problem of accelerator physics, mathematics theory of dynamical systems, and other fields of physics. The focus is on the appearance of chaos in a beam distribution. A study of the problem is based on two observation: First, Using Lyapunov method and its extension we obtain solutions of partial differential equations $[1,2]$. Using this approach we discuss the problem of finding a solution of Vlasov-Poisson equation, i.e., some stationary solution where we consider magnetic field as some disturbance with a small parameter. Thus the solution of Vlasov equation yields an asymptotic series such that the solution of Vlasov-Poisson equation is the basis solution for one. The second observation is that physical chaos is weakly limit of, well known, the Landau bifurcation's. This fact we have proved using ideas on the Nature of Turbulence [3].
\end{abstract}

\section{INTRODUCTION}

The aim of this paper is to find the solution for VlasovPoisson (VP) equation in the attraction region of an integral manifold. As known VP is system which can be written as follows

$$
\begin{gathered}
\partial_{t} f+v \partial_{x} f+E(t, x) \partial_{v} f=0 \\
\Delta U(t, x)=4 \pi \gamma \rho(t, x)
\end{gathered}
$$

Where

$$
\begin{gathered}
E(t, x):=-\partial_{x} U(t, x) ; \\
\rho(t, x):=\int f(t, x, v) d v .
\end{gathered}
$$

Here we have $\gamma=+1, f=f(t, x, v)$ is the distribution of the particles in phase space depending upon the time $t \geq 0$, the position $x \in R^{n}$ and the velocity, $U=U(t, x)$ is the Newtonian potential . The quantity $n$ indicates the space dimension. If not stated otherwise, we assume $n=3$. With the opposite sign of $\gamma$, i.e. for $\gamma=-1$, the VP system is used as a first

\footnotetext{
* Supported by U.S. Department of Energy contract number DE-AC02. $98 \mathrm{CH} 10886$.

$\dagger$ E-mail: parsa@bnl.gov

‡ E-mail: zvf@umex.istrada.net.ua
}

model to describe the evolution of equally charged particles (with repulsive forces) in plasma physics.

As well known, this system is considered to be an approximation to the more involved Vlasov-Maxwell system [1]. Below we shall reduce the quasi linear equation $(1,2)$ to a linear partial differential equation of first order.

\section{LANDAU BIFURCATION AND WEAKLY- CONVERGENCE}

Using the arguments in [4], we get

$$
f(t, x, v)=f_{0}(x, v) e^{-i \omega t} .
$$

Thus, the function $f_{0}(x, v)$ satisfies equation

$$
\partial_{x} f_{0}+\partial_{v} f_{0} E=i \omega f_{0}
$$

Let

$$
\rho_{0}=\int f_{0}(x, v) d v
$$

then the coefficients of the equation be independent of the time $t$. A general solution $f(t, x, v)$ may be introduced by a sum of the particular solutions such that the function $f$ depends on the time by the multiplier of the type $e^{-i \omega t}$. Here the frequency $\omega$ can't be arbitrary. They be defined completely from the equation (6). The equation (6) is quasi linear equation. Using the well known approach, we reduce it to a linear equation by the way of introducing some function:

$$
w=-f_{0}+f_{0}(x, v)=0
$$

then

$$
L w \equiv \partial w_{x} v+\partial w_{v} E=\partial w_{f_{0}}\left(i \omega f_{0}\right)
$$

The equation (9) is a linear equation.

In the following, we transform (9) into a Fredholm's equation

$$
w=R f_{0} .
$$

Where $R$ is some kernel operator, i.e.

$$
\sum\left|\lambda_{i}\right|<\infty, \lambda_{i} \in \sigma(R),
$$


here $\sigma(R)$ is spectrum of $R$ (see [1],[4]). Now, by differentiating equation (10) with respect to the given VP system, we obtain

$$
f_{0}=\mu \tilde{R} f_{0}
$$

where $\tilde{R}=L R$, and $\mu=-i \omega^{-1}$. Suppose there exists a small bounded set of data $(x, v)$ such that $\tilde{R}_{0}$ is a small operator:

$$
|\mu|<\operatorname{mes}\left|\Omega_{N}\right||| \tilde{R}_{1} \|^{-1} .
$$

We assume that the measure of the domain $\Omega_{N}$ increases with increasing number $N$, and $\omega \rightarrow 0$. This case yields to some bifurcation sequence. One may be the divergent series or the convergent series. The term "the convergent" may have the various sense.

Theorem: Under the assumption $\left|f_{0 N}\right| \leq 1$ for all $N$, there is an invariant measure $\tau_{0}$ such that $\lim _{N \rightarrow \infty} d \tau_{N} \equiv$ $f_{0 N} d x=d \tau^{0}$. This measure be a stochastic measure on the manifold $f_{0}(x, v)=1$. Thus we have an ergodic motion on this manifold. As opposed to first case, we have a wave motion.

Consequently the Landau chaotization take place for series bifurcation is not always true.

\section{REFERENCES}

[1] Zubov V.I., Integral Equations for the Lyapunov Function. Soviet Math. Dokl. Vol. 42(1991), N 2, p. 535-537.

[2] Parsa Z., Zadorozhny V.F., On the beam dynamics which has been described by Vlasov Equation. Proceedings of the fifth international workshop: Beam dynamics \& optimization. St. Petersburg, Russia,1998.

[3] Rueller D., Takens F., On the Nature of Turbulence, common. Math. Phys.,20,167-192, Springer- Verlag,1971.

[4] Parsa Z., Zadorozhny V.F., On the solution of Vlasov-Poisson equation in some neighborhood of a singular point (to appear). 